\title{
Post COVID Syndrome: A Challenge to Address
}

\author{
Harshal Tukaram Pandve ${ }^{1, *}$ and Sarika Prashant Patil ${ }^{2}$ \\ ${ }^{1}$ Department of Community Medicine (PSM), Pimpri Chinchwad Municipal Corporation's Yashwantrao Chavan Memorial Hospital \& Postgraduate \\ Medical Institute, Pimpri, Pune Maharashtra, India
}

${ }^{2}$ Department of Community, Medicine, SBH Government Medical College, Dhule, Maharashtra, India

*Corresponding author: Harshal Tukaram Pandve, Professor and Head, Department of Community Medicine (PSM), Pimpri Chinchwad Municipal Corporation's Yashwantrao Chavan Memorial Hospital \& Postgraduate Medical Institute, Pimpri, Pune Maharashtra, India, E-mail: dr_harshalpandve@yahoo.co.in

Received: 18 Dec, 2020 | Accepted: 28 Dec, 2020 | Published: 31 Dec, 2020

Citation: Pandve HT, Patil SP (2020) Post COVID Syndrome: A Challenge to Address. J Epidemiol Public Health Rev 5(4): dx.doi. org/10.16966/2471-8211.204

Copyright: (C) 2020 Pandve HT, et al. This is an open-access article distributed under the terms of the Creative Commons Attribution License, which permits unrestricted use, distribution, and reproduction in any medium, provided the original author and source are credited.

This editorial article discusses briefly about the Post COVID Syndrome, its challenges and also some solutions to address it.

Global impact of COVID-19 has shattered the world starting from devastating health issues and extending to wellbeing of mankind. First case of Novel Corona virus was detected in China in November 2019 and COVID 19 was declared as pandemic by World Health Organization (WHO) in March 2020. Varied mitigation strategies and management protocols had been tested and adopted all over the world. Novel Corona virus disease pandemic caused by severe acute respiratory syndrome coronavirus 2 (SARS CoV-2) had varied presentation affecting multiple systems in human.

While fighting against the battle of global pandemic COVID-19, we all are learning more about the impact of the disease on immediate health as well as on long term physical and mental health too [1].

Acute management of the illness may affect to future systemic abnormalities. The road of recovery and recovered one has wide range of health impacts and also having range of illness affecting the afterlife of COVID-19 recovered patients.

The researchers should emphasize on regular follow-up surveys of COVID-19 recovered patients who are in the convalescent phase so that it would be helpful to evaluate any changes in the acquired immune function, blood parameters, psychological factors, biochemical factors, lung, brain, eye, kidney, heart and Gastrointestinal (GI) tract functions over the course of time. By knowing the possible complications of its after-effect from the recovered patients will be helpful to ascertain the future disease complications and provides more information for the development of vaccines and drugs for these kinds of pandemics in the future [2].

The strains of human corona viruses like severe acute respiratory syndrome (SARS-CoV), Middle East respiratory syndrome (MERS$\mathrm{CoV}$ ) and Severe acute respiratory syndrome coronavirus 2 (SARSCoV-2) having similarities in clinical manifestation with potential severe symptoms. Report show that $30 \%$ of people with SARS or Middle East respiratory syndrome had persisting lung abnormalities after their acute illness [3]. Interstitial lung disease and pulmonary vascular disease are likely to be the most important respiratory complications [4]. Cardiovascular complications of influenza infection, including myocarditis, acute myocardial infarction, and exacerbation of heart failure have been well-recognized during previous historical epidemics and make a significant contribution to mortality [5]. The association between the novel Coronavirus and cardiac complications needs further exploration and clinicians should be aware of the potential impact of cardiovascular conditions and complications in COVID-19 patients, which should require more extensive and frequent monitoring [6]. It has been evident that there are Cardiovascular implications of fatal outcomes in patients with COVID-19. The Cerebro-vascular involvement of post COVID complication has also predominantly emerged as a fatal outcome after the pandemic. With increasing incidence of stroke the recovery state and recovered state after COVID-19 has been of due importance. Stroke was one of the early or delayed presentations of multiorgan involvement of COVID-19 infected young patients due to rupture or leak of blood vessels or even ischemia [7]. Just like other entities with acute cardiac injury, there is likely to be a diverse response, depending on the mechanism of myocardial injury, severity of acute illness, therapy delivered, hemodynamic response, host factors, immunemediated factors, and post recovery care and follow-up [8].

Post-COVID recovery clinics are initiated for patients with persistent health issues due to the viral infection or acute health events such as stroke/myocardial infarction or chronic conditions like hypertension/diabetes etc. Also to name few of the post COVID issues by some patients facing Autonomic function alteration, reduced endurance, impaired renal functions, myopathies, polyneuropathy, residual pulmonary manifestations, psychological issues, myocardial injuries or progression of chronic issue [9]. Identification by screening of systemic involvement in convalescent phase show acute recovery from the primary corona infection but may have subclinical or delayed systemic complication. As the COVID-19 pandemic has progressed, there has been growing awareness of the long-term impacts of COVID-19 infection, including cardiac, neurological, 
metabolic and respiratory long-term sequelae. Also, some evidence shows that olfactory dysfunction and gustatory dysfunction are relatively common long-term sequelae associated with COVID-19 infection [10].

Post recovery from COVID 19, the patients should be vigilant about their health status and follow-up. Sometimes symptoms still persist months after the infection suggests the presence of a 'post-COVID-19 syndrome' [9].

\section{Post- COVID-19 holistic assessment includes}

1. Assessment and management of breathlessness, oxygen requirements and dysfunctional breathing, postviral cough.

2. Full pulmonary function testing.

3. Walk test with an assessment of oxygen saturation.

4. Consideration of a new diagnosis of venous thromboembolic disease.

5. Echocardiogram.

6. Consideration of specific post-intensive care unit complications such as sarcopaenia, cognitive impairment and post- traumatic stress disorder.

7. Psychosocial and anxiety assessment and onward referral where required.

8. Consideration of rehabilitation needs and onward referral where required.

Another important issue which has to be addressed is the side-effect of medication interruption due to COVID-19 for elderly with chronic diseases as COVID-19 mortality rate among elderly with chronic diseases is higher than for other age groups. It is important to ensure mitigation risk of COVID-19 virus infecting the elderly who visit the hospital for refill prescription; it also has an impact on whether patients are willing to come back to the hospital where they got the prescription for long-term treatment. For reducing the impact of COVID-19 on elderly with chronic diseases and mitigating risk of prescription drugs shortage, Hsiao SH, et al., [11] study had suggested to set up outdoor dispensary counters for drug receiving without need to enter hospital should be one of the requisite measures for drug dispensing services during epidemics.

To address the emerging long term consequences for patients recovering from COVID-19 there is urgent need of combined efforts from policy makers and health care professionals.

As rehabilitation is envisaged to come from existing health services, occupational therapy, physiotherapy, primary care, dentistry, mental health services, dieticians and speech and language therapists will be in demand that may outstrip resources available [12].

To conclude with, post COVID-19 is as important challenge to address as COVID-19 disease itself. A multipronged and multidisciplinary approach is required to handle it effectively.

\section{References}

1. PHOSP-COVID (2020) Improving long-term health outcomes. University of Leicester, England.

2. Chen N, Zhou M, Dong X, Qu J, Gong F, et al. (2020) Epidemiological and clinical characteristics of 99 cases of 2019 novel coronavirus pneumonia in Wuhan, China: a descriptive study. Lancet 395: 507513.

3. Bandyopadhyay D, Akhtar T, Hajra A, Gupta M, Das A, et al. (2020) COVID-19 Pandemic: Cardiovascular Complications and Future Implications. Am J Cardiovasc Drugs 20: 311-324.

4. George PM, Barratt SL, Condliffe R, Desai SR, Devaraj A, et al. (2020) Respiratory follow-up of patients with COVID-19 pneumonia. Thorax 75: 1009-1016.

5. Xiong T-Y, Redwood S, Prendergast B, Chen M (2020) Coronaviruses and the cardiovascular system: acute and long-term implications. Eur Heart J 41: 1798-1800.

6. Sabatino J, De Rosa S, Di Salvo G, Indolfi C (2020) Impact of cardiovascular risk profile on COVID-19 outcome. A meta-analysis. PLoS One 15: e0237131.

7. Mehta S, Samdani MN (2020) Shadow of COVID-associated stroke lingers over recovered patients. The Times of India, India.

8. Mitrani RD, Dabas N, Goldberger JJ (2020) COVID-19 cardiac injury: Implications for long-term surveillance and outcomes in survivors. Heart Rhythm 17: 1984-1990.

9. Penn Medicine (2020) Recovery After COVID-19: Assessing the Long-Term Effects of SARS-CoV-2. Penn Medicine, USA.

10. Ontario Agency for Health Protection and Promotion (Public Health Ontario) (2020) Long-Term Sequelae and COVID-19-What We Know So Far. Toronto, ON: Queen's Printer for Ontario1-12.

11. Hsiao S-H, Chang H-M, Kang Y-T, Chen Y-H (2020) Mitigate risks of medication interruption due to COVID-19 for elderly with chronic diseases. Kaohsiung J Med Sci 36: 661-662.

12. Croner-i (2020) After-care needs of post-Covid survivors. Croner-i, UK. 